\title{
Focal neuroendocrine carcinoma mixed with adenocarcinoma of the gallbladder with aggressive lymph node metastasis in a patient who did not meet the mixed neuroendocrine-non-neuroendocrine neoplasm criteria
}

\author{
Yoshie Kadota $^{1,2} \circledast$. Shinsuke Funakoshi ${ }^{3}$. Shigemichi Hirose ${ }^{4}$. Eisuke Shiomi ${ }^{5} \cdot$ Masanori Odaira $^{1}$. \\ Haruka Yagishita ${ }^{1} \cdot$ Yosuke Kobayashi $^{1} \cdot$ Fumiki Toriumi $^{1}$ - Seiichi Tamai ${ }^{4} \cdot$ Takashi Endo $^{1} \cdot$ Hirohisa Harada ${ }^{1}$
}

Received: 17 June 2021 / Accepted: 26 October 2021 / Published online: 12 November 2021

(c) The Author(s) 2021

\begin{abstract}
A 70-year-old Japanese woman who was treated for interstitial pneumonia (IP) with steroid therapy developed cholecystitis. A serial computed-tomography (CT) imaging showed irregular thickness of the fundus wall of the gallbladder and two rapidly enlarged lymph nodes (LNs): number (no.) 12 and no. 8a. Positron-emission tomography-computed tomography (PET-CT) scan showed an abnormal uptake at the site of the gallbladder tumor and those LNs. We subsequently performed open radical cholecystectomy and LN dissection of the no. 12 and 8a LNs, following complete remission of IP. The histology showed gallbladder adenocarcinoma, with a single focus of neuroendocrine carcinoma (NEC) component of less than 30\%; Ki-67 index $>80 \%$, synaptophysin (Syn) (+), chromogranin A (CgA) (+), and clusters of differentiation (CD) $56(+)(\mathrm{T} 2 \mathrm{bN} 1 \mathrm{M} 0$, Stage IIIB). LN no. 8a was diffusely metastatic with NEC components. LN no. 12c, which was adjacent to the cystic duct, revealed necrosis without apparent tumor cells, but was highly suspicious for tumor necrosis. The final diagnosis was adenocarcinoma of the gallbladder with focal NEC $(<30 \%)$, which did not meet the criteria for mixed neuroendocrine-nonneuroendocrine neoplasm (MiNEN). Postoperatively, she completed 4 cycles of adjuvant chemotherapy for NEC (Cisplatin plus Etoposide), and no recurrence was observed after 12 months.
\end{abstract}

Keywords Gallbladder · Adenocarcinoma - Neuroendocrine carcinoma $\cdot$ Mixed adenoneuroendocrine carcinoma $($ MANEC) $\cdot$ Mixed neuroendocrine-non-neuroendocrine neoplasm $($ MiNEN) $\cdot$ Lymph node metastasis

\section{Introduction}

Yoshie Kadota

yoshiekadota@gmail.com

1 Department of General and Gastrointestinal Surgery, Tokyo Saiseikai Central Hospital, 1-4-17 Mita, Minato-ku, Tokyo 108-0073, Japan

2 Department of Surgery, Nippon Koukan Hospital, 1-2-1 Kokandori, Kawasaki Ward, Kawasaki, Kanagawa 210-0852, Japan

3 Department of Medical Oncology, Tokyo Saiseikai Central Hospital, 1-4-17 Mita, Minato-ku, Tokyo 108-0073, Japan

4 Department of Diagnostic Pathology, Tokyo Saiseikai Central Hospital, 1-4-17 Mita, Minato-ku, Tokyo 108-0073, Japan

5 Department of Radiology, Tokyo Saiseikai Central Hospital, 1-4-17 Mita, Minato-ku, Tokyo 108-0073, Japan
The occurrence of gallbladder neuroendocrine carcinoma (NEC) is very rare, which is reported to be $2.2 \%$ of all gallbladder cancers, which usually presents with advanced stage, lymph nodes (LNs) metastases, and poor prognosis [1]. More than a third of gallbladder NEC is mixed with a non-neuroendocrine component, most frequently, adenocarcinoma [2, 3]. According to the 2019 World Health Organization (WHO) Classification, these mixed tumors are called mixed neuroendocrine-non-neuroendocrine neoplasm (MiNEN) (previously known as MANEC: mixed adenoneuroendocrine carcinoma), if both components are present in more than $30 \%$ of the tumor [4]. In addition, neuroendocrine tumor (NET) Grade (G) 3 and NEC are categorized separately in the new classification. Well-differentiated NET is now NET G3, and poorly differentiated NET is considered 
NEC, both with a mitotic rate (mitoses $\left./ 2 \mathrm{~mm}^{2}\right)>20$, or Ki-67>20\% [4]. The median overall survival of biliary NEC and MiNEN are reported as 9.6 and 12.2 months, respectively [5]. Because gallbladder MiNEN is a rare tumor, the overall survival has not been well investigated. Furthermore, the true nature of how aggressive the tumor progresses have not been well reported. In this case report, while the patient was treated for interstitial pneumonia (IP), we were able to follow the rapid progression of the gallbladder adenocarcinoma with focal NEC $(<30 \%)$ and LN metastasis, over the course of 4 months with serial computed-tomography (CT) imaging. This was a unique case where we were able to observe the behavior of this aggressive tumor, while waiting for the complete remission of IP before performing surgical intervention.

\section{Case report}

A 70-year-old woman was referred to our surgical division by her pulmonologist who was treating her for idiopathic IP for 1 year prior to presentation, for an incidental finding of chronic cholecystitis found on the CT scan. Three months prior to referral, she was started on steroid therapy for exacerbation of her IP, which was tapered down to Prednisolone (PSL) $12.5 \mathrm{mg} /$ day at the time of consultation. The patient reported recurring mild episodes of right subcostal pain, which she did not seek medical attention for. Her past medical history was unremarkable except for childhood asthma and untreated uterine myoma. Most notably, her past surgical history was significant for endoscopic mucosal resection (EMR) for a $4 \mathrm{~mm}$ NET G1 of the lower rectum, 20 months prior to initial consultation. Other past surgical history included an appendectomy for appendicitis in childhood, and a left partial oophorectomy for two benign ovarian cysts. She was not taking any medications except for PSL $12.5 \mathrm{mg}$, which was tapered down over 1 month from the initial dosing of $30 \mathrm{mg}$. Her family history was non-contributory. She reported no known drug allergies. Her social history included smoking one pack (20 cigarettes) per day for 40 years, which she recently quit, but she denied drinking alcohol or use of recreational drugs. She is a retired desk worker. Laboratory data were within normal limits as shown in Table S1, including carcinoembryonic antigen (CEA) and cancer antigen 19-9 (CA19-9).

Initial CT showed signs of cholecystitis with a mild and diffuse wall thickening with multiple small gall stones (Fig. 1). A follow-up CT scan, which was performed 2 months later, revealed irregular thickening of the gallbladder fundus wall in addition to two enlarged LNs; number (no.) 12 and no.8a, $1.5 \mathrm{~cm}$ and $0.7 \mathrm{~cm}$, respectively. Even though malignancy was suspected at this point, because she was still on $10 \mathrm{mg}$ of PSL, surgical intervention was postponed. Around the same time, she was treated with a single dose of $50 \mathrm{mg}$ of Azathioprine by her pulmonologist. Subsequent CT scan at 4 months showed persistent irregular wall thickening of the gallbladder fundus. Furthermore, no. $12 \mathrm{LN}$ increased in its size to $1.8 \mathrm{~cm}$ from $1.5 \mathrm{~cm}$, and no. $8 \mathrm{a} \mathrm{LN}$ increased in its size to $1.8 \mathrm{~cm}$ from $0.7 \mathrm{~cm}$. Both LNs emerged and enlarged rapidly, presenting with non-homogenous low densities within the LNs, suspicious for necrosis (Fig. 1). On the Positron-emission tomography-computed tomography (PET-CT) scan, an abnormal uptake of fluorodeoxyglucose $\left({ }^{18} \mathrm{~F}-\mathrm{FDG}\right)$ were observed in the fundus of the gallbladder, as well as no. 12 and no. 8a LNs (Fig. 2). In addition, T1-, T2-, and diffusion-weighted magnetic resonance imaging (MRI) was performed. The findings were notable for high intensity observed in the fundus of the gallbladder, as well as LNs no. 12 and no. 8a (Fig. S1).

Serial CT scan, MRI, and PET-CT scan all pointed to the diagnosis of the gallbladder tumor with $\mathrm{LN}$ metastases. She underwent radical cholecystectomy with liver-wedge resection and $\mathrm{LN}$ dissection, 5 months after the initial surgical consultation. The $\mathrm{LN}$ dissection included all hot spots seen on the PET-CT scan, including the no. 12, no. 8a, and no. 13a lymphatic basins. No liver metastases or peritoneal dissemination were found. Intraoperative ultrasound showed no infiltration to the liver bed, and therefore, no further hepatectomy was recommended. Careful excision of the enlarged LN no. 8a revealed significantly fragile LN, indicating potential metastasis. Intraoperative frozen section of the cystic duct was negative for tumor cells. The postoperative course was uneventful except for mildly symptomatic delayed gastric emptying, which is not unusual in patients who are treated with hepatoduodenal ligament dissection. She was discharged home from the hospital on postoperative day 17 .

Histology of the gallbladder showed well-to-moderately differentiated adenocarcinoma, according to the UICC 8th edition), with a focal NEC component $(<30 \%)$, which was positive for synaptophysin (Syn), chromogranin A $(\mathrm{CgA})$, and clusters of differentiation (CD) 56, and Ki-67 index $>80 \%$ on immunohistochemistry (T2bN1M0 Stage IIIB, according to the UICC 8th edition) (Fig. 3). Based on these findings, the tumor was diagnosed as gallbladder adenocarcinoma with NEC, which did not meet the criteria for MiNEN. LN no. 8a was diffusely metastatic with NEC components, which were positive for Syn, CgA, and CD 56 , and was the only LN that showed clear metastasis based on histology (Fig. 4). The LN no. 12c, which was adjacent to the cystic duct was predominantly necrotic without apparent tumor cells, but was highly suspicious for tumor necrosis. Because the histology of the $\mathrm{LN}$ revealed diffusely metastatic NEC, she received 4 cycles of adjuvant chemotherapy with Cisplatin $\left(80 \mathrm{mg} / \mathrm{m}^{2}\right)$ on day 1 and Etoposide $\left(100 \mathrm{mg} / \mathrm{m}^{2}\right)$ on days $1-3$, every 4 weeks, beginning 1 month 


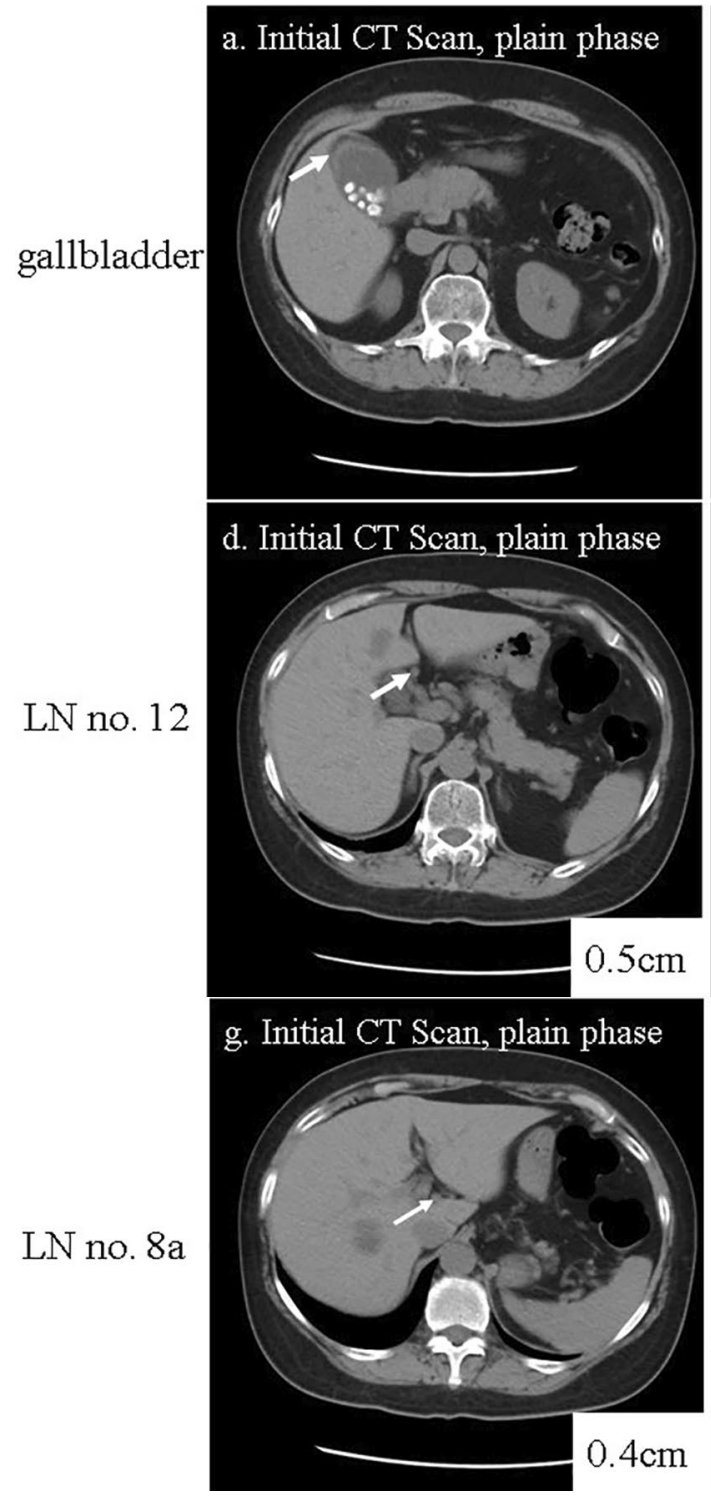

Fig. 1 Preoperative serial CT scans of the gallbladder. The figure shows preoperative serial CT scans showing rapid tumor growth in the gallbladder and the adjacent LNs, over the course of 4 months. $\mathbf{a}, \mathbf{d}, \mathbf{g}$ CT scan at the time of surgical consultation. b, e, $\mathbf{h}$ Follow-up CT scan, 2 months later. c, f, i Follow-up CT scan, 4 months later.

postoperatively. No recurrence was observed for 12 months, at the time of this study.

\section{Discussion}

Gallbladder MiNENs are extremely rare. Costa et al. [6] published a literature review in 2021, and reported that there are only 24 cases of gallbladder MiNEN (which used to be called MANEC) including this case, reported across the literature. Although our case did not meet the criteria for MiNEN [4], this report supports our understanding of how
Initial CT scan showed no apparent tumors in the gallbladder or the LNs (a, d, g). CT scan at 2 and 4 months revealed gradual thickening of the fundus wall of the gallbladder $(\mathbf{b}, \mathbf{c})$, rapid growth of the LN no. $12(\mathbf{e}, \mathbf{f})$, and the LN no. $8 \mathrm{a}(\mathbf{h}, \mathbf{i})$. No apparent liver infiltration or peritoneal dissemination were observed

rare and aggressive the gallbladder MiNEN is, and therefore, close postoperative surveillance is critical.

In MiNEN, NEC components are known to show more aggressive patterns $[3,7]$. Generally, in MiNEN, aggressiveness and prognosis are driven by the most aggressive neoplastic components [8,7]. Furthermore, MiNEN with predominant NEC components are more likely to metastasize to LNs [3]. Several authors reported that patients with resectable cases of MiNEN who underwent complete surgical resection had overall better prognosis $[2,8,9]$. Chen et al. [1] described the aggressive nature of the gallbladder NEC, and reported that patients with NEC had significantly 
gallbladder

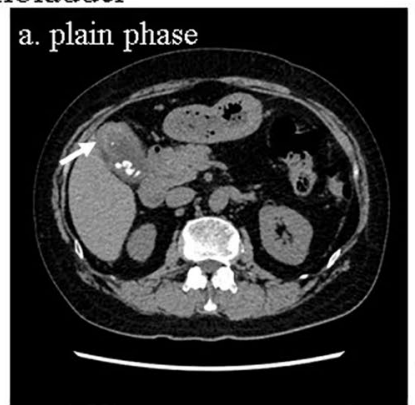

LN no. 12

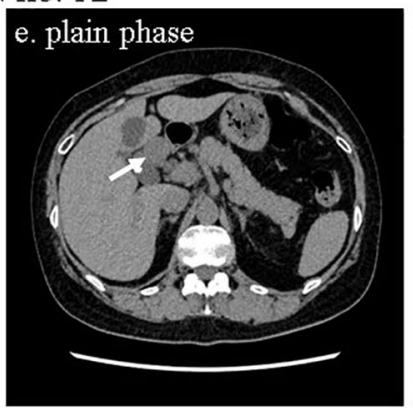

LN no. 8a

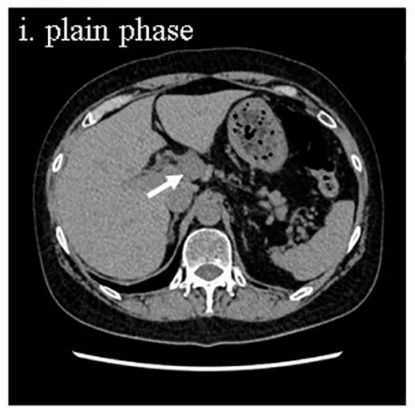

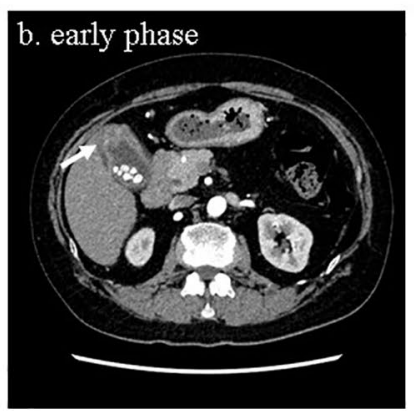
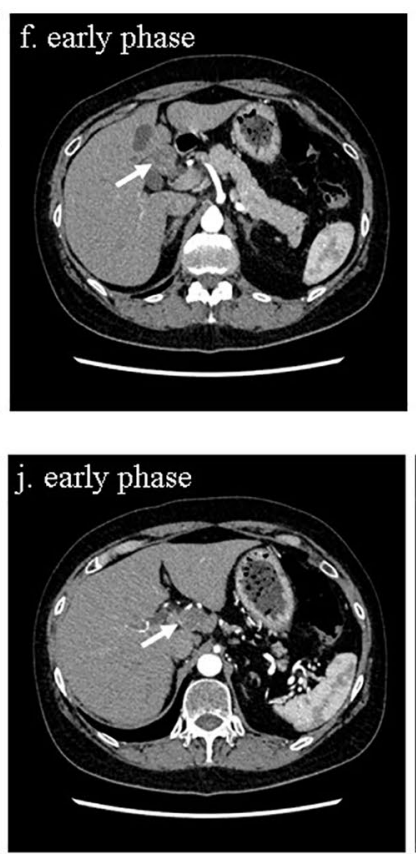
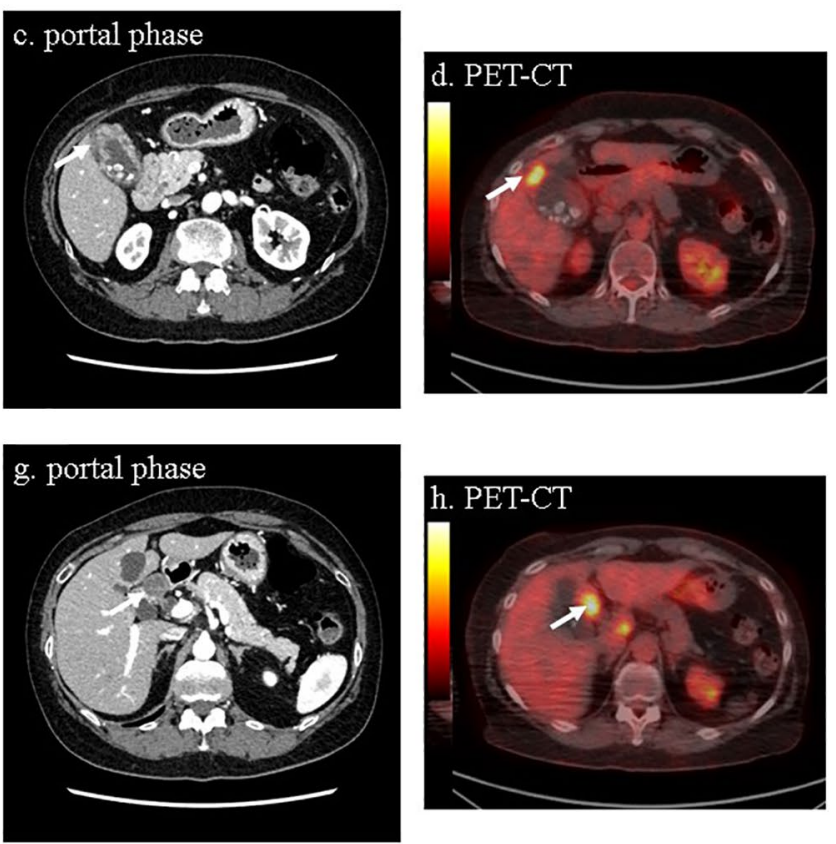

Fig. 2 Dynamic CT scan and PET-CT scan findings of the gallbladder. The figure shows dynamic CT scan and PET-CT scan results, 4 months later, taken at the same time as Fig. 1c, f, and i, preoperatively. The irregularly thickened wall showed an increased density in the fundus of the gallbladder on CT scan $(\mathbf{a}-\mathbf{c})$. In the LNs No.

higher rate of $\mathrm{LN}$ metastases and poorer prognosis, when compared to patients with gallbladder adenocarcinoma. In their study, median survival time was found to be longer in patients who underwent surgical resection, chemotherapy, and radiation therapy, when compared to patients who received palliative resection of the gallbladder cancer.

This is a unique case report where we were able to observe the behavior of this aggressive tumor, while waiting for the complete remission of IP before performing surgical intervention, which took place 5 months after the initial diagnosis of cholecystitis. Consequently, the serial CT scan demonstrated the tumor rapidly emerging and progressing in just 4 months, especially in the no. 8a LN.

Glucocorticoid PSL is known for its anti-inflammatory response in many chronic inflammatory diseases. It is also widely used as part of various cancer treatments. In our
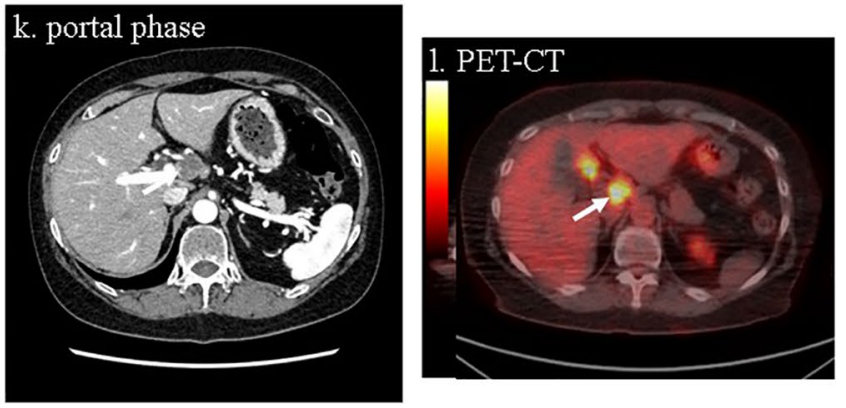

12 and $8 \mathrm{a}$, the cortex of the LNs were slightly enhanced, with nonhomogenous contrast enhancement seen within both LNs $(\mathbf{e}-\mathbf{g}, \mathbf{i}-\mathbf{k})$. PET-CT scan showed increased ${ }^{18}$ F-FDG uptake in the fundus of the gallbladder, LNs no. 12, and no. 8a (d, h, l)

patient, the oral PSL and the single dose Azathioprine administration may have contributed to the rapid progression of the gallbladder adenocarcinoma with NEC. However, the effects of immunosuppressive therapies on the tumor progression remain unknown [10]. Further studies are warranted.

If neoadjuvant chemotherapy is considered, an endoscopic-ultrasonography-guided-fine-needle aspiration (EUS-FNA) should be considered unless there are no contraindications. However, in hindsight, EUS-FNA likely would have not yielded the correct diagnosis in this patient, because the gallbladder NEC was small. Furthermore, majority of the providers biopsy one representing $\mathrm{LN}$ to rule out metastasis. In this patient, unless LN no. 8a was selected over LN no. 12c by chance, NEC components may have been missed. 


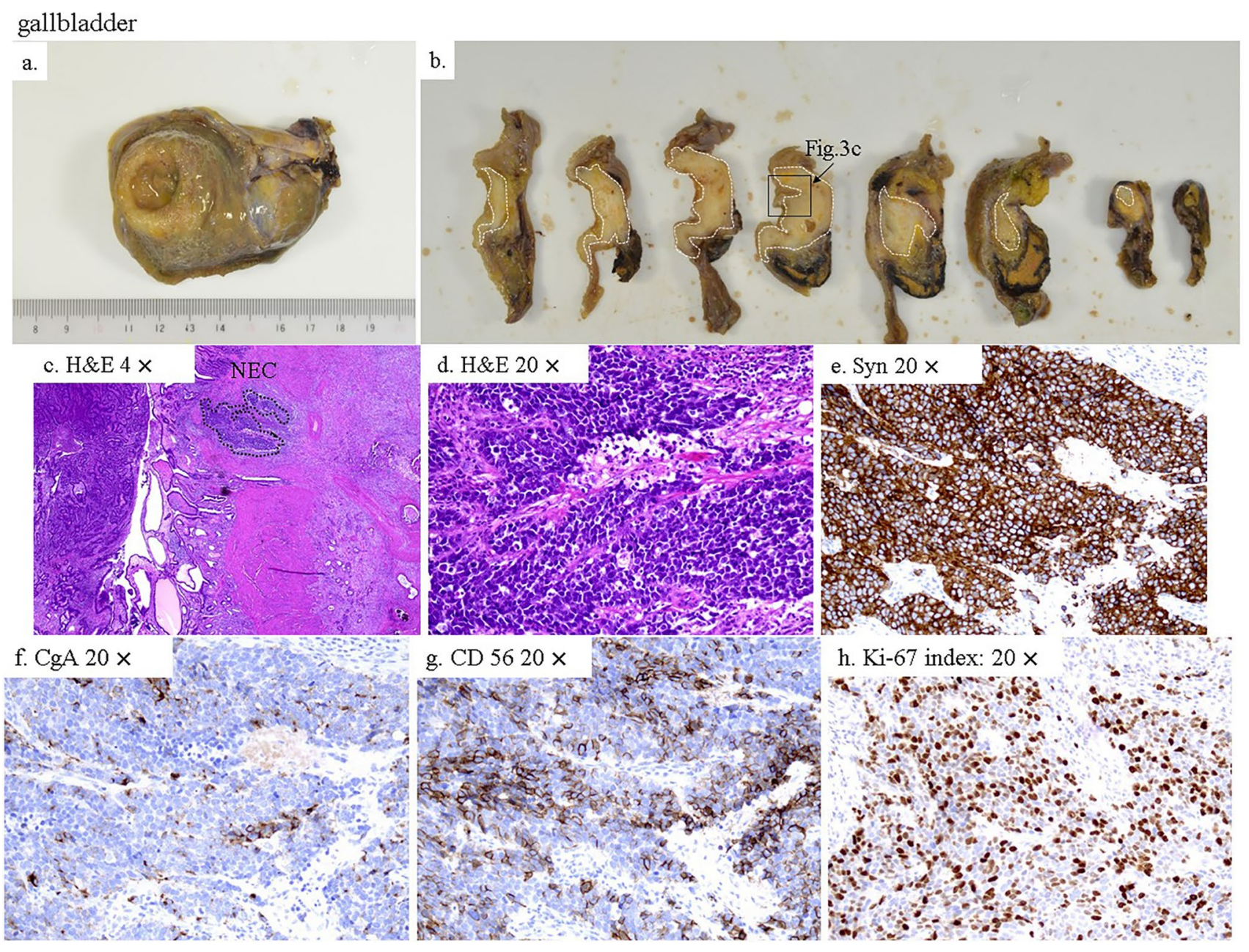

Fig. 3 Histopathological analysis of the gallbladder. a the gross specimen of the surgically excised gallbladder. $\mathbf{b}$ axial sections of the gallbladder shown in a. The neoplasm in the fundus was diagnosed as well-to-moderately differentiated adenocarcinoma with focal NEC components (c, d), which were diffusely positive for Syn (e), while

Okada et al. [11] reported a similar case of an aggressive focal gallbladder NEC mixed with adenocarcinoma which did not meet the criteria for MiNEN. In their report, the patient was surgically treated with subsequent adjuvant chemotherapy, successfully. However, 4 months later, the patient presented with multiple metastases of NEC in the para-aortic LNs. Despite undergoing a second round of chemotherapy, the patient expired 8 months after the initial surgery. These cases highlight how aggressive the NEC components behave, regardless of the size of the primary NEC. Due to the aggressive nature of NEC components with frequent LN involvement, we highly recommend performing the standard recommended dissection of the hepatoduodenal ligament, no. 12 , no. 8a, and no. 13a LNs, based on our experience combined with other previous reports $[1,3,5$, $11]$.
CgA (f) and CD56 (g) were focally positive, and Ki-67 was over $80 \%$ (h) (T2bN1M0, Stage IIIB, according to the UICC 8th edition). $H \& E$ hematoxylin and eosin staining, Syn synaptophysin, $C g A$ chromogranin A, CD 56 clusters of differentiation, $N E C$ neuroendocrine carcinoma, $\times 20 \times 20$ magnification

There is currently no consensus on the postoperative adjuvant chemotherapy, but it is usually determined based on the most aggressive components of MiNEN [8, 7, 12]. The latest National Comprehensive Cancer Network (NCCN) guideline recommends several therapy options for resectable gallbladder cancer, including; (a) observation without any additional therapy, (b) neoadjuvant/adjuvant systemic chemotherapy, and (c) fluoropyrimidine + radiotherapy (RT) [13]. On the other hand, NCCN guideline recommends several therapy options for resectable extrapulmonary NEC, including (a) resection + adjuvant chemotherapy $+/-\mathrm{RT}$, (b) neoadjuvant chemotherapy $+/-\mathrm{RT}+$ resection, (c) chemotherapy alone, (d) RT alone, and (e) definitive chemoradiation with Cisplatin + Etoposide (or Carboplatin + Etoposide) [14]. In our patient, the most aggressive component was determined to be NEC, as supported by LN metastases. Therefore, the patient subsequently received 4 cycles of Cisplatin $(80 \mathrm{mg} /$ 

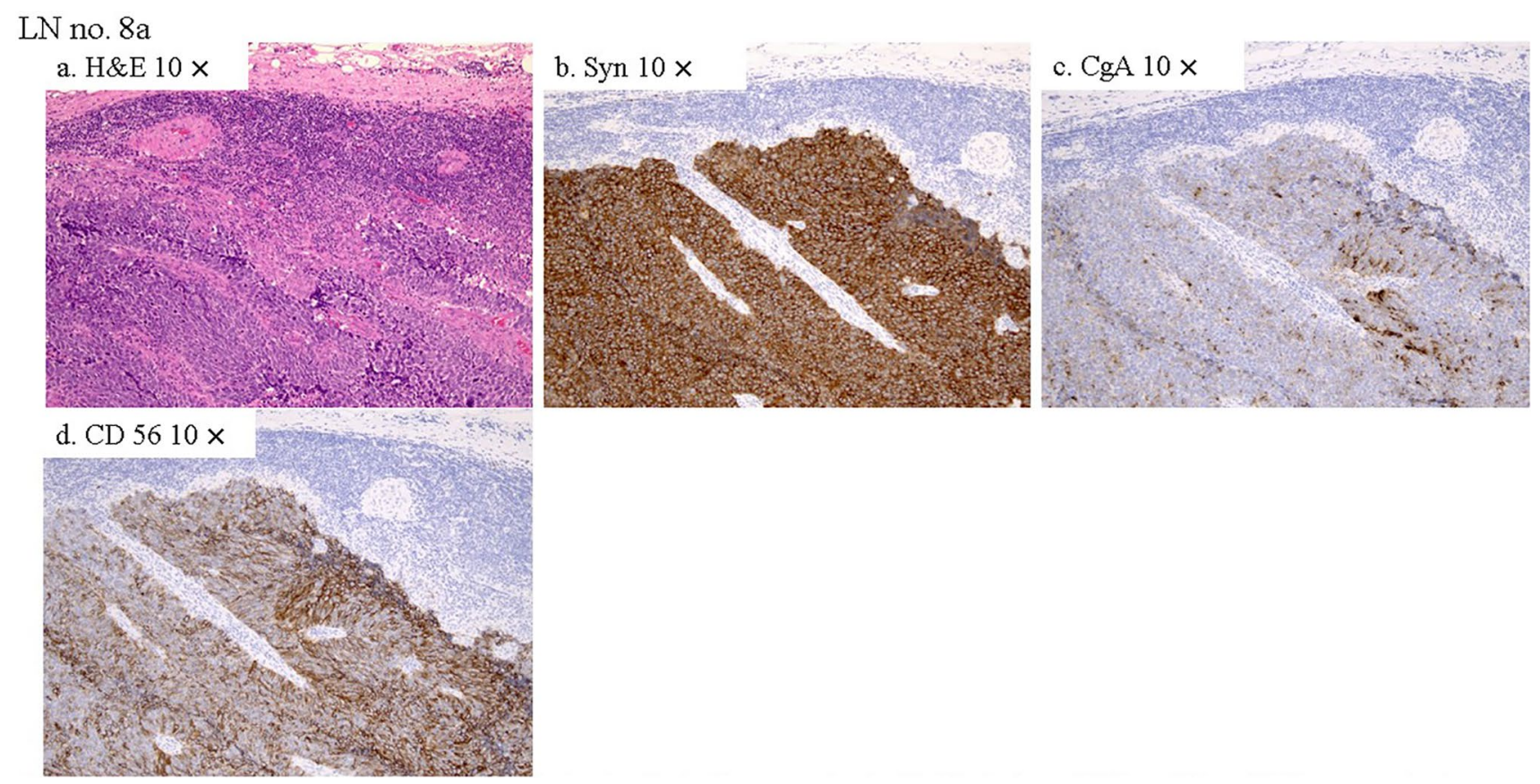

Fig. 4 Histopathological analysis of the LN 8a. Metastases were only detected in LN No. 8a (a) which was completely occupied by highly proliferative NEC cells, which were positive for Syn (b), CgA (c), and CD 56 (d), same as the gallbladder NEC components (Fig. 3e-g).

$\mathrm{m}^{2}$ ) on day 1 and Etoposide $\left(100 \mathrm{mg} / \mathrm{m}^{2}\right)$ on days $1-3$, every 4 weeks.

At the time of this study which was 12 months after total cholecystectomy with LN dissection, no recurrence of the tumor was observed. If the predominant component of the MiNEN is NEC, the tumor tends to behave more aggressively, presenting with frequent LN metastases [3, 7]. Gallbladder NEC is notorious for high recurrence rate, poor morbidity, and mortality $[1,5,11,12]$. The patient will continue to be closely monitored.

This patient underwent EMR for a lower rectum G1 NET, 2 years prior to this procedure. The previous tumor was a different tumor, which was negative for Syn, CgA, CD56, and Ki-67 on immunohistochemistry, in contrast to NEC components of this tumor.

Although each component of MiNEN must account for at least $30 \%$ of the whole neoplasm to meet the criteria for MiNEN, several authors suggested that the prognosis was influenced by the most aggressive histological components, regardless of the percentage of those components $[8,9,15]$. Our patient had less than $30 \%$ of NEC components, who did not meet the criteria for MiNEN, but presented with diffusely metastatic LNs with NEC components. Because NEC components were the most aggressive histological components, our patient was successfully treated with adjuvant chemotherapy for NEC,
$H \& E$ hematoxylin and eosin staining, Syn synaptophysin, $C g A$ chromogranin A, CD 56 clusters of differentiation, NEC neuroendocrine carcinoma, $\times 10 \times 10$ magnification

with no recurrence observed at 12 months follow-up, at the time of this study. Our patient may raise a question challenging the current $30 \%$ cut off as defined by the WHO 5th edition. In our patient, the tumor had less than $30 \%$ of NEC components which did not satisfy the criteria for MiNEN, but behaved very aggressively with early LN metastases. Therefore, aggressive treatment including surgical intervention and adjuvant chemotherapy may be necessary for patients presenting with any percentage $(<30 \%)$ of NEC components that behaves like MiNEN $(>30 \%)$.

In conclusion, our case highlights the fact that the gallbladder adenocarcinoma with NEC $(<30 \%)$ which did not meet the criteria for MiNEN, may rapidly progress in just a few months with LN metastases, driven by NEC components. Radical surgery combined with adjuvant chemotherapy (4 cycles of Cisplatin with Etoposide) showed good results over this highly aggressive tumor. Based on our limited experience, for patients with gallbladder adenocarcinoma with NEC, we highly recommend performing cholecystectomy with further LN dissection of the LNs no. 12 , no. $8 \mathrm{a}$, and no. 13a, even if it does not meet the criteria for MiNEN. Further investigation is warranted.

Supplementary Information The online version contains supplementary material available at https://doi.org/10.1007/s12328-021-01547-8. 
Acknowledgements We are grateful to Dr. Emi Saegusa-Beecroft for writing assistance.

\section{Declarations}

Conflict of interest Yoshie Kadota, Shinsuke Funakoshi, Shigemichi Hirose, Eisuke Shiomi, Masanori Odaira, Haruka Yagishita, Yosuke Kobayashi, Fumiki Toriumi, Seiichi Tamai, Takashi Endo, and Hirohisa Harada declare that they have no conflict of interest.

Human rights All procedures followed have been performed in accordance with the ethical standards laid down in the 1964 Declaration of Helsinki and its later amendments.

Informed consent Informed consent was obtained from all patients for being included in the study.

Open Access This article is licensed under a Creative Commons Attribution 4.0 International License, which permits use, sharing, adaptation, distribution and reproduction in any medium or format, as long as you give appropriate credit to the original author(s) and the source, provide a link to the Creative Commons licence, and indicate if changes were made. The images or other third party material in this article are included in the article's Creative Commons licence, unless indicated otherwise in a credit line to the material. If material is not included in the article's Creative Commons licence and your intended use is not permitted by statutory regulation or exceeds the permitted use, you will need to obtain permission directly from the copyright holder. To view a copy of this licence, visit http://creativecommons.org/licenses/by/4.0/.

\section{References}

1. Chen C, Wang L, Liu X, et al. Gallbladder neuroendocrine carcinoma: report of 10 cases and comparision of clinicopathologic features with gallbladder adenocarcinoma. Int J Clin Exp Pathol. 2015;8:8218-26.

2. Adsay NV, La Rosa S. Neuroendocrine neoplasms of the gallbladder and bile ducts. In: WHO Classification of Tumours Editorial Board, editor. Digestive system tumours. Lyon: International Agency for Research on Cancer; 2019. p. 292-4.

3. Harada K, Sato Y, Ikeda H, et al. Clinicopathologic study of mixed adenoneuroendocrine carcinomas of hepatobiliary organs. Virchows Arch. 2012;460:281-9.

4. Klimstra DS, Klöppel G, La Rosa S, et al. Classification of neuroendocrine neoplasms of the digestive system. In: WHO classification of tumours editorial board. Digestive system tumours.
Lyon: International Agency for Research on Cancer; 2019. p. $16-9$.

5. Kim J, Lee WJ, Lee SH, et al. Clinical features of 20 patients with curatively resected biliary neuroendocrine tumours. Dig Liver Dis. 2011;43:965-70.

6. Costa AC, Cavalcanti CLC, Coelho HGB, et al. Rare mixed adenoneuroendocrine carcinoma of the gallbladder: case report and review of literature. Am J Case Rep. 2021;22: e929511.

7. Machairas N, Paspala A, Frountzas M, et al. Mixed adenoneuroendocrine carcinoma (MANEC) of the gallbladder: a systematic review of outcomes following surgical management. In Vivo. 2019;33:1721-6.

8. de Mestier L, Cros J, Neuzillet C, et al. Digestive system mixed neuroendocrine-non-neuroendocrine neoplasms. Neuroendocrinology. 2017;105:412-25.

9. Wen LJ, Chen JH, Xu HJ, et al. The clinical profiles, management, and prognostic factors of biliary mixed neuroendocrine nonneuroendocrine neoplasms: a systematic review of the literature. Medicine (Baltimore). 2020;99: e23271.

10. Mohammadi L, Mosayyebi B, Imani M, et al. Correlation between dexamethasone and miRNAs in the regulation of apoptosis, drugresistance, and metastasis of cancer cell. Curr Mol Med. 2020. https://doi.org/10.2174/1566524020666200925155614.

11. Okada H, Uchida Y, Matsuzaki N, et al. A case of neuroendocrine carcinoma in the hepatic hilar lymph nodes concomitant with an adenocarcinoma of the gallbladder. World J Surg Oncol. 2016;14:284.

12. Kamei K, Shindoh J, Kiya Y, et al. Conversion surgery after extensive chemotherapy for stage IV mixed adenoneuroendocrine carcinoma (MANEC) of the gallbladder: clinical implications from the patterns of response and recurrence. Clin J Gastroenterol. 2020;13:240-6.

13. Benson AB, D'Angelica MI, Abbott DE, et al. Biliary tract cancers: gallbladder cancer. In: NCCN clinical practice guidelines in oncology. hepatobiliary cancers (Version 5.2021). https://www. nccn.org/professionals/physician_gls/pdf/hepatobiliary.pdf. Accessed 21 Sept 2021.

14. Ettinger DS, Wood DE, Aggarwal C, et al. NCCN guidelines insights: non-small cell lung cancer, version 1.2020. J Natl Compr Canc Netw. 2019;17:1464-72.

15. Frizziero M, Chakrabarty B, Nagy B, et al. Mixed neuroendocrine non-neuroendocrine neoplasms: a systematic review of a controversial and underestimated diagnosis. J Clin Med. 2020;9:273.

Publisher's Note Springer Nature remains neutral with regard to jurisdictional claims in published maps and institutional affiliations. 